\title{
Testing a Low Molecular Mass Fraction of a Mushroom (Lentinus edodes) Extract Formulated as an Oral Rinse in a Cohort of Volunteers
}

\author{
Caterina Signoretto, ${ }^{1}$ Gloria Burlacchini, ${ }^{1}$ Anna Marchi, ${ }^{1}$ Marcello Grillenzoni, ${ }^{2}$ \\ Giacomo Cavalleri, ${ }^{2}$ Lena Ciric, ${ }^{3}$ Peter Lingström, ${ }^{4}$ Elisabetta Pezzati, ${ }^{1,5}$ Maria Daglia, ${ }^{6}$ \\ Egija Zaura, ${ }^{7}$ Jonathan Pratten, ${ }^{3}$ David A. Spratt, ${ }^{3}$ Michael Wilson, ${ }^{3}$ and Pietro Canepari ${ }^{1}$ \\ ${ }^{1}$ Sezione di Microbiologia, Dipartimento di Patologia e Diagnostica, Università di Verona, Strada Le Grazie 8, 37134 Verona, Italy \\ ${ }^{2}$ Sezione di Chirurgia Maxillo Facciale e di Odontostomatologia, Dipartimento di Chirurgia, Università di Verona, \\ Piazzale A. Scuro 10, 37134 Verona, Italy \\ ${ }^{3}$ Division of Microbial Diseases, UCL Eastman Dental Institute, 256 Gray's Inn Road, London WC1X 8LD, UK \\ ${ }^{4}$ Department of Cariology, Institute of Odontology at Sahlgrenska Academy, University of Gothenburg, Box 450, \\ 40530 Göteborg, Sweden \\ ${ }^{5}$ DIPTERIS, Università di Genova, Corso Europa 26, 16132 Genova, Italy \\ ${ }^{6}$ Dipartmento di Chimica Farmaceutica, Università di Pavia, Via Taramelli 12, 27100 Pavia, Italy \\ ${ }^{7}$ Department of Preventive Dentistry, Academic Centre for Dentistry Amsterdam (ACTA), Gustav Mahlerlaan 3004, \\ 1081 LA Amsterdam, The Netherlands
}

Correspondence should be addressed to Pietro Canepari, pietro.canepari@univr.it

Received 15 June 2011; Accepted 11 July 2011

Academic Editor: Carla Pruzzo

Copyright (c) 2011 Caterina Signoretto et al. This is an open access article distributed under the Creative Commons Attribution License, which permits unrestricted use, distribution, and reproduction in any medium, provided the original work is properly cited.

\begin{abstract}
Although foods are considered enhancing factors for dental caries and periodontitis, laboratory researches indicate that several foods and beverages contain components endowed with antimicrobial and antiplaque activities. A low molecular mass (LMM) fraction of an aqueous mushroom extract has been found to exert these activities in in vitro experiments against potential oral pathogens. We therefore conducted a clinical trial in which we tested an LMM fraction of shiitake mushroom extract formulated in a mouthrinse in 30 young volunteers, comparing the results with those obtained in two identical cohorts, one of which received water (placebo) and the other Listerine. Plaque index, gingival index and bacterial counts in plaque samples were determined in all volunteers over the 11 days of the clinical trial. Statistically significant differences $(P<0.05)$ were obtained for the plaque index on day 12 in subjects treated with mushroom versus placebo, while for the gingival index significant differences were found for both mushroom versus placebo and mushroom versus Listerine. Decreases in total bacterial counts and in counts of specific oral pathogens were observed for both mushroom extract and Listerine in comparison with placebo. The data suggest that a mushroom extract may prove beneficial in controlling dental caries and/or gingivitis/periodontitis.
\end{abstract}

\section{Introduction}

Dental plaque is a complex bacterial biofilm associated with tooth and gum surfaces $[1,2]$. The so-called "plaquedependent" oral pathologies include dental caries and periodontitis, with the former being the result of acid destruction of the tooth enamel and dentin caused mainly by Streptococcus mutans in the presence of sucrose [3], while the latter results from host aggression to the structure supporting the teeth by a bacterial consortium, consisting mainly of strict anaerobes [4]. Although foods, often in association with poor oral hygiene, are considered enhancing factors for these pathologies, a substantial amount of laboratory research indicates that several foods and beverages of vegetal origin contain components endowed with antimicrobial, antiadhesive, and antiplaque activities (for a recent review 
see Signoretto et al. [5]). Thus, the consumption of such healthy foods and beverages could be encouraged or foods may be enriched with the active component(s). Several epidemiological studies have been conducted in distinct populations (Tibetans, Israeli Arabs, Senegalese, and Japanese, English and US schoolchildren), comparing tea drinkers versus nontea drinkers; the results showed a lower incidence of caries in tea-drinkers [6, 7]. In addition, a significant reduction in both saliva and dental plaque counts of mutans streptococci and lactobacilli has been observed, together with a reduced plaque score in adult tea, coffee, and wine drinkers versus water drinkers [8]. Very recently it has been shown that the regular consumption of foods enriched with these active molecules (wine and coffee) is associated with a modification of the oral microbial community in the direction of less periodontopathogenic microbiota [9]. In order to support the use of these substances as mouthrinses or toothpastes in daily oral hygiene, a few clinical studies have been conducted with interesting, though not conclusive, results. Tea and tea extracts $[10,11]$ and a high molecular mass cranberry constituent [12] in mouthrinse formulations have been tested in volunteers. A low molecular mass (LMM) fraction of an aqueous mushroom extract (Lentinus edodes, commonly called shiitake and very popular in Japan) has been found to exert antimicrobial, antiadhesive, and antiplaque activities in in vitro experiments against several potential oral pathogens $[13,14]$. We therefore conducted a clinical trial in which we tested an LMM fraction of a shiitake extract formulated in a mouthrinse in 30 young volunteers, comparing the results with those obtained in two identical cohorts, one of which received coloured and aromatised water (placebo) and the other Listerine, a mouthwash used worldwide.

\section{Materials and Methods}

2.1. Composition of Mouthrinses. Three different mouthrinses were tested in this study. LMM fractions of the mushroom extract mouthrinse as well as a mouthrinse acting as a negative control (placebo) and composed of aromatised water alone were prepared by MicroPharm Ltd, Newcastle Emyln, UK. The aromatised water contained 12\% ethanol, 6\% glycerine, $0.2 \%$ PEG 40 Hydrogenated Castor Oil (Cremophor RH40), 0.2\% flavour, and 0.05\% saccharin. The LMM fraction of the mushroom extract mouthrinse was obtained as described elsewhere [15] and added to the aromatised water at a final concentration of $2 \mathrm{x}$ in comparison with the original food concentration. Twenty $\mathrm{mL}$ of both mouthrinses were dispensed in $25 \mathrm{~mL}$ tubes and maintained at $4^{\circ} \mathrm{C}$ in the dark for no more than 5 months. "Freshburst" Listerine (Johnson and Johnson, New Brunswick, NJ, USA) was the one we tested as a positive control. Listerine contained the following active ingredients: $0.092 \%$ eucalyptol, $0.042 \%$ mentol, $0.06 \%$ methyl salicylate and $0.064 \%$ thymol in water, alcohol $(21.6 \%)$, sorbitol solution, flavoring, poloxamer 407, benzoic acid, sodium saccharin, sodium benzoate, D\&C yellow no. 10, and FD\&C green no. 3. Listerine was removed from the original bottle and $20 \mathrm{~mL}$ aliquots were poured into tubes identical to those of the other mouthrinses.

2.2. Volunteers Enrolled. A total of 90 healthy volunteers (49 males and 41 females, average age 22, range 19-27 years) from the student population of the University of Verona were included in a three-leg, double-blind, clinical trial. Exclusion criteria included antibiotic treatment at any time in the 3 months prior to the study, oral soft tissue pathologies, missing teeth, visible untreated carious lesions, smoking, and pregnancy. Subjects were randomly assigned to the experimental group in which mushroom was tested $(n=30)$, to the negative/placebo (water) control group $(n=30)$ and to the positive (Listerine) control $(n=30)$. All volunteers were asked to sign an informed consent form.

2.3. Study Design. This clinical trial was authorised by the Ethical Committee of the Verona University Hospital, reference 1653 dated March 11, 2009. A three-leg, doubleblind study was carried out using the three mouthrinses twice daily for 12 days. The complete experimental period was 18 days (Figure 1). All participants were examined by a dentist and subjected to professional oral hygiene treatment at time 0 . On days 1 to 6 , all subjects were invited to maintain regular oral hygiene (brushing and flossing) at home. On day 6 a sample of dental plaque was collected along with clinical measurements, as described below, and each participant received the assigned mouthwash. From day 7 to day 17, they were instructed to refrain from any type of oral hygiene and to perform only two rinses with $10 \mathrm{~mL}$ of mouthwash for $30 \mathrm{sec}$ at a $1 \mathrm{~min}$ interval twice daily (morning and evening). They were asked to refrain from eating and drinking 1 hour after rinsing. On days $8,10,12$, and 17 , a subgingival plaque sample was collected from each participant. Finally, on day 18 all the volunteers were subjected to professional oral hygiene to restore oral health.

2.4. Plaque Index (PI) and Gingival Index (GI) Determination. The following measurements were performed on days 6,8 , 10,12 , and 17 of the study: (i) plaque index (PI) or plaque accumulation was evaluated on the buccal surfaces following the application of disclosing solution (Butler GUM, Butler, Chicago, IL, USA), according to the Turesky modification of the Quigley-Hein plaque index [16]; (ii) gingival index or gingival inflammation was assessed on the buccal and lingual marginal gingivae and the interdental papillae, according to the Loe-Silness gingivitis index [17].

2.5. Sample Collection for Microbiological Analysis. Dental plaque samples were collected using a curette from two upper molar surfaces on the left and right sides. Plaque was dispersed in $750 \mu \mathrm{L}$ sterile Tris-EDTA buffer $(10 \mathrm{mM}$ TrisHCL, 1 mM EDTA, pH 8.0), vortexed for 1 min to uniformly disperse the plaque, subdivided into three aliquots and stored at $-80^{\circ} \mathrm{C}$ until further use. 


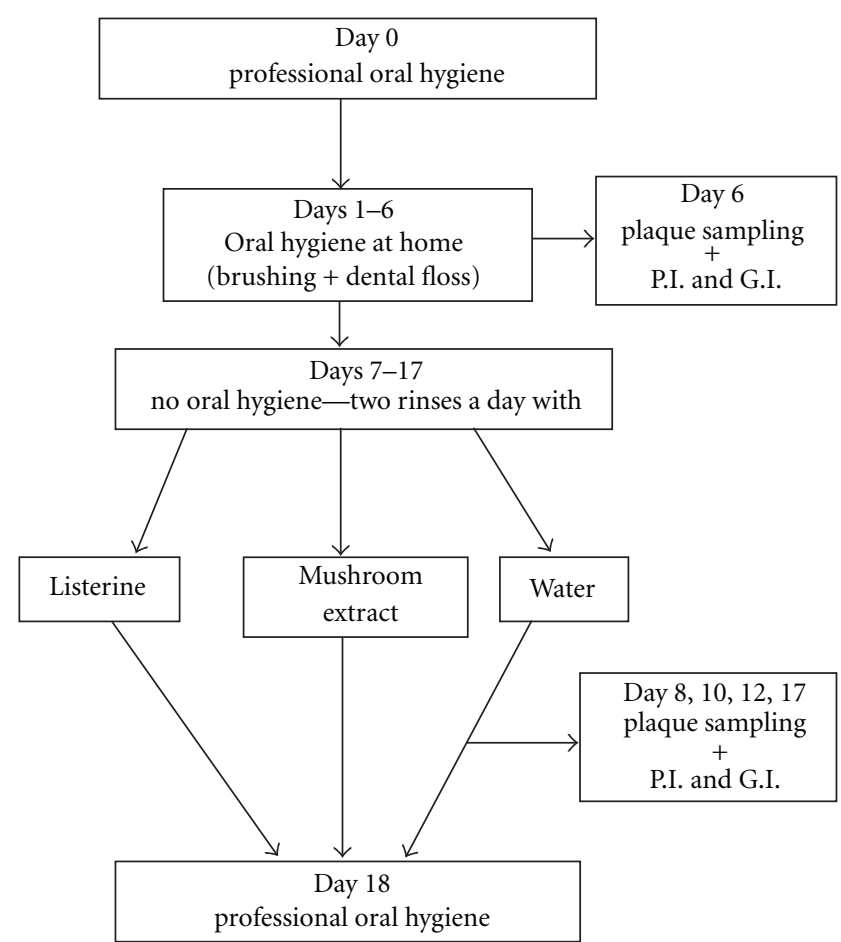

Figure 1: Outline of the study. PI, plaque index; GI, gingival index.

2.6. Quantitative PCR ( $q P C R$ ). DNA was extracted from plaque biofilms using a phenol: chloroform : iso-amyl alcohol (25:24:1) bead-beating extraction method [18], which involves physical cell lysis, protein removal, and finally DNA precipitation using polyethylene glycol. Three triplex qPCR assays were then carried out using $2 \mu \mathrm{L}$ of extracted DNA to quantify eight oral taxa as well as the total number of organisms. The taxa investigated were Fusobacterium nucleatum, Lactobacillus casei, Veillonella dispar, Neisseria subflava, Actinomyces naeslundii, Prevotella intermedia, Streptococcus sanguinis, and Streptococcus mutans. The assays were performed using the Rotor-Gene 6500 (QIAGEN) instrument and Sensimix Probe (Bioline) qPCR mix according to the manufacturers' instructions using previously published oligonucleotide sequences [19].

2.7. Statistical Evaluation. Data collected were analysed by using the Statistical Package for Social Sciences (SPSS version 16.0). For each mouthwash, the mean and standard deviation of the values was calculated. Data were analysed using oneway analysis of variance (ANOVA) followed by Turesky test for pairwise comparison. $P$ values $<0.05$ were considered statistically significant.

\section{Results}

3.1. Clinical Evaluation of the Effects of the Three Mouthrinses. All 90 volunteers included in the three groups, one of which received the mushroom mouthrinse, the second coloured aromatised water (placebo), and the third Listerine, completed the study. At the beginning and on another four
Table 1: Cumulative plaque index scores ( \pm standard deviation) at the various test sessions during the treatment of volunteers with three different mouthwashes. Statistically significant differences $(P<0.05)$ are marked by $*$.

\begin{tabular}{lccc}
\hline Test session & Mushroom & Water & Listerine \\
\hline Day 6 & $0.24 \pm 1.61$ & $0.17 \pm 0.12$ & $0.19 \pm 0.18$ \\
Day 8 & $0.49 \pm 0.26$ & $0.62 \pm 0.36$ & $0.63 \pm 0.34$ \\
Day 10 & $0.84 \pm 0.38$ & $0.93 \pm 0.42$ & $1.01 \pm 0.39$ \\
Day 12 & $0.83 \pm 0.30^{*}$ & $1.04 \pm 0.41^{*}$ & $0.91 \pm 0.38$ \\
Day 17 & $0.95 \pm 0.36$ & $1.05 \pm 0.38$ & $0.93 \pm 0.34$ \\
\hline
\end{tabular}

TABLE 2: Cumulative gingival index scores ( \pm standard deviation) at the various test sessions during the treatment of volunteers with three different mouthwashes. Statistically significant differences $(P<0.05)$ are marked by $*$ or $\neq$.

\begin{tabular}{lccc}
\hline Test session & Mushroom & Water & Listerine \\
\hline Day 6 & $0.20 \pm 0.20$ & $0.15 \pm 0.15$ & $0.22 \pm 0.28$ \\
Day 8 & $0.26 \pm 0.18$ & $0.31 \pm 0.25$ & $0.27 \pm 0.20$ \\
Day 10 & $0.40 \pm 0.26$ & $0.42 \pm 0.24$ & $0.46 \pm 0.22$ \\
Day 12 & $0.53 \pm 0.30^{* \ddagger}$ & $0.70 \pm 0.34^{*}$ & $0.69 \pm 0.32^{\ddagger}$ \\
Day 17 & $0.56 \pm 0.31$ & $0.65 \pm 0.33$ & $0.59 \pm 0.31$ \\
\hline
\end{tabular}

days of the period of mouthrinse use (Figure 1), clinical parameters such as PI and GI were determined for all volunteers. Table 1 shows the results of the PI calculation of mean values \pm SD. Mean values increased over time in the three mouthrinse categories, but, as expected, the placebo group showed the greatest increase. Significant differences $(P<0.05)$ were observed on day 12 regarding mushroom treatment versus placebo. A similar pattern was observed when GI was evaluated (Table 2). GI values increased with time, but, again, higher values were observed in the placebo group in comparison with both the mushroom and Listerine mouthrinses. Statistically significant differences $(P<0.05)$ were obtained on day 12 for both mushroom versus placebo and mushroom versus Listerine treatment.

3.2. Microbiological Measurement of the Effects of the Three Mouthrinses. At the time-points above indicated and also at the time of collection of the clinical data, plaque samples were obtained in order to determine the load of total bacteria and of selected oral microorganisms by quantitative PCR (pPCR). We quantified four microorganisms associated with gingivitis (A. naeslundii, F. nucleatum, P. intermedia, L. casei), one strongly implicated in dental caries (S. mutans), and three microorganisms associated with oral health ( $S$. sanguinis, $N$. subflava, $V$. dispar). The eight oral microorganisms amounted to about $23 \pm 6 \%$ of the total microbiota of the dental plaque. Figure 2 shows the evolution of the four microorganisms associated with gingivitis in comparison with the total count during treatment with the three distinct mouthrinses. In the placebo group, the mean total cell count values increased over time (from $1.48( \pm 1.63) \times 10^{7}$ on day 6 to $2.27( \pm 0.99) \times 10^{8}$ on day 17$)$. Standard deviation is presented in brackets. Individual counts were F. nucleatum 


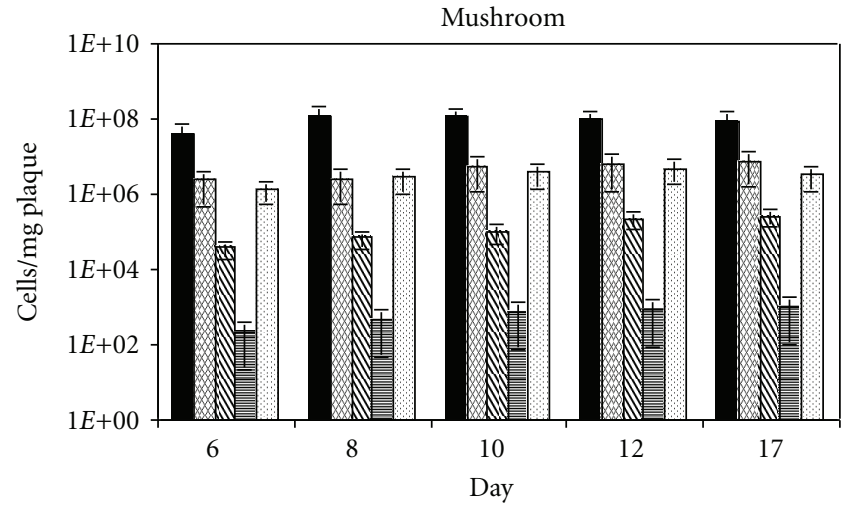

(a)

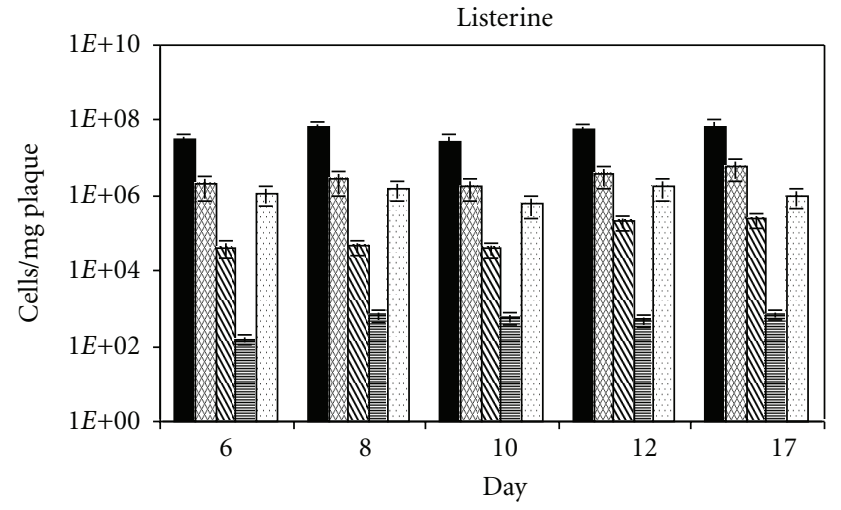

(b)

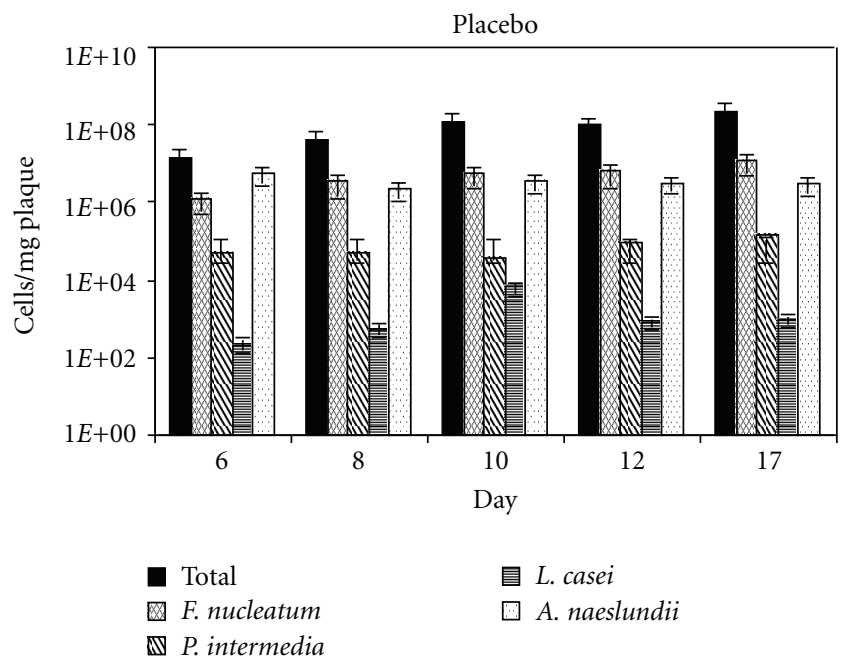

(c)

FIGURE 2: Numbers of each of the investigated taxa associated with gingivitis and total counts in dental plaque samples of the three cohorts enrolled in the study. Standard error bars represent the standard deviation $(n=30)$.

(from $1.28( \pm 1.85) \times 10^{6}$ to $\left.1.24( \pm 0.76) \times 10^{7}\right)$, P. intermedia (from $4.89( \pm 10.3) \times 10^{4}$ to $\left.4.53( \pm 3.25) \times 10^{5}\right)$, and $A$. naeslundii (from $5.48( \pm 8.35) \times 10^{5}$ to $2.99( \pm 3.72) \times 10^{6}$ on days 6 and 17, resp.) increased in accordance with the total count, while $L$. casei, after an initial increase on day 10 , tended to decrease (from $2.20( \pm 2.86) \times 10^{2}$ on day 6 to $6.43( \pm 2.86) \times 10^{3}$ on day 10 to $9.34( \pm 4.09) \times 10^{2}$ on day 16). The oral rinses with mushroom or Listerine in general did not block the increase either in total counts or in those of the four gingivitis-associated microorganisms, but these increases never exceeded half the final value reported for placebo. In detail, F. nucleatum during treatment with mushroom increased from $2.23( \pm 2.95) \times 10^{6}$ on day 6 to $7.56( \pm 4.28) \times 10^{6}$ on day 17 , and from $1.93( \pm 3.05) \times 10^{6}$ to $5.74( \pm 3.57) \times 10^{6}$ during treatment with Listerine; $P$. intermedia from $3.71( \pm 7.72) \times 10^{4}$ to $2.60( \pm 6.66) \times 10^{5}$ with mushroom and from $4.26( \pm 10.7) \times 10^{4}$ to $2.46( \pm 2.69) \times 10^{5}$ with Listerine treatment; $A$. naeslundii from $1.41( \pm 2.27) \times$ $10^{6}$ to $3.30( \pm 5.48) \times 10^{6}$ and from $1.15( \pm 2.03) \times 10^{6}$ to $9.70( \pm 10.4) \times 10^{5}$; and $L$. casei from $2.23( \pm 2.91) \times$ $10^{2}$ to $9.28( \pm 2.76) \times 10^{2}$ and from $1.57( \pm 1.98) \times 10^{2}$ to $5.13( \pm 1.75) \times 10^{2}$ after treatment with mushroom and Listerine, respectively. Figure 3 shows the effects of the three mouthrinses tested on the cariogenic S. mutans. In the placebo group, the $S$. mutans cell count increased over time (from $4.17( \pm 9.94) \times 10^{4}$ to $\left.4.57( \pm 2.67) \times 10^{5}\right)$ on day 17. Although both mushroom and Listerine induced cell number increases, the final $S$. mutans values were lower than those of the placebo group (i.e., $2.15( \pm 5.58) \times 10^{5}$ and $3.68(1.15) \times 10^{5}$ for mushroom and Listerine, resp.). Finally, Figure 4 shows the effects of the three mouthrinses on three microorganisms associated with oral health. $S$. sanguinis, $N$. subflava, $V$. dispar counts increased (up to 2 $\log$ ) over time from day 6 to day 17 in both the placebo and mushroom groups. The values obtained were $S$. sanguinis from $1.60( \pm 2.26) \times 10^{5}$ to $3.92( \pm 1.03) \times 10^{6}$ for placebo and from $4.32( \pm 5.62) \times 10^{5}$ to $2.53( \pm 0.62) \times 10^{6}$ for mushroom group; N. subflava from $7.04( \pm 8.52) \times 10^{5}$ to $4.48( \pm 0.45) \times$ $10^{7}$ and from $7.14( \pm 8.48) \times 10^{5}$ to $9.49( \pm 2.60) \times 10^{6}$ for placebo and mushroom, respectively; $V$. dispar from $1.31( \pm 2.36) \times 10^{6}$ to $2.10( \pm 0.88) \times 10^{7}$ and $2.48( \pm 3.28) \times$ $10^{6}$ to $1.37( \pm 0.31) \times 10^{7}$ for placebo and mushroom, 


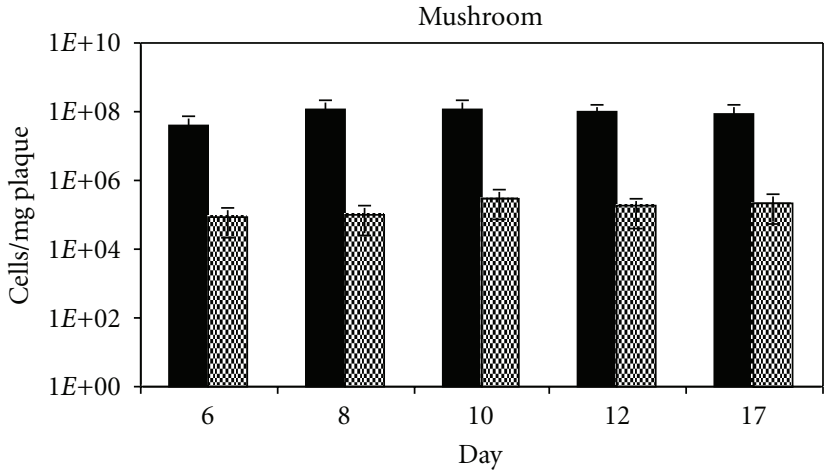

(a)

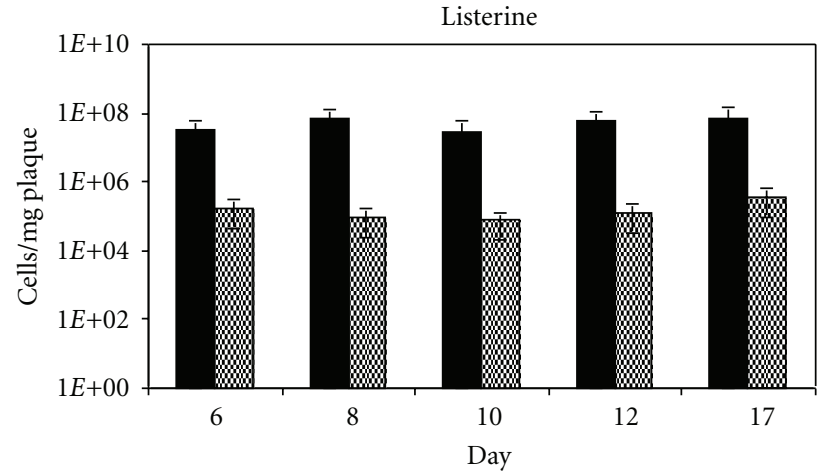

(b)

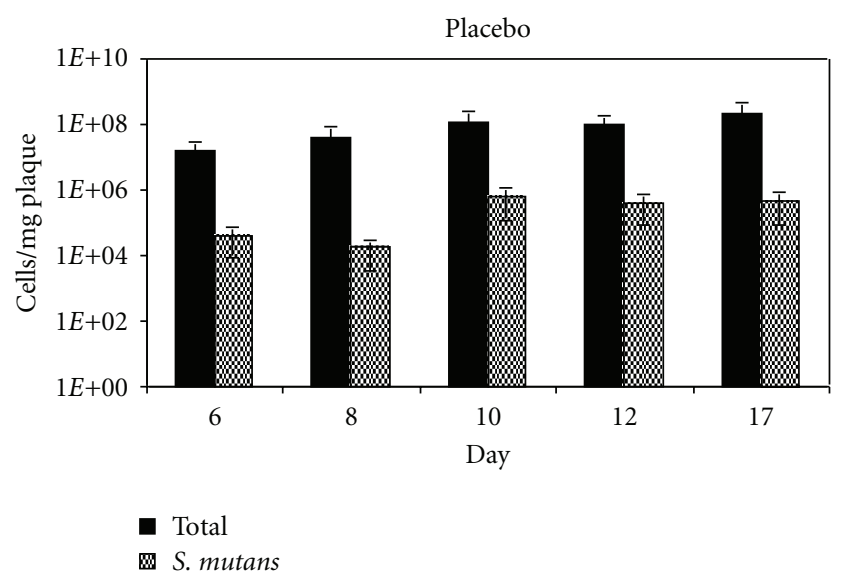

(c)

FIgURE 3: Numbers of $S$. mutans and total counts in dental plaque samples of the three cohorts enrolled in the study. Standard error bars represent the standard deviation $(n=30)$.

respectively. Listerine, on the contrary, prevented any cell number increase of $N$. subflava and $V$. dispar and, thus values at day 17 were quite close to the initial (day 6) count (i.e., $2.95( \pm 3.71) \times 10^{6}$ and $3.67( \pm 4.41) \times 10^{6}$, resp. $)$. The cell number increase of $S$. sanguinis was only partially affected by Listerine (from $1.87( \pm 3.40) \times 10^{5}$ to $1.51( \pm 3.20) \times$ $\left.10^{6}\right)$. Although differences were seen, especially as far as the placebo group versus mushroom or Listerine was concerned, none of them proved statistically significant.

\section{Discussion}

The aim of this clinical trial was to evaluate the possible beneficial effects of an LMM mushroom extract formulated as a mouthrinse in preventing dental plaque deposition in volunteers. In addition, its activity was compared with the activity of Listerine, a widely used mouthwash and with that of a placebo composed of colored and aromatised water. The rationale of this study was based on the proven in vitro activity of this mushroom extract in terms of antimicrobial, antiadhesive, antiplaque and plaque disaggregating activity against several oral microorganisms including those involved either in dental caries or in gingivitis/periodontitis [13].

The results on the whole indicate that a mouthrinse containing an LMM fraction of mushroom extract was capa- ble of significantly reducing dental plaque deposition as compared with placebo, but this activity was not significantly higher than that displayed by Listerine. Worthy of note, however, is the fact that in the present clinical trial Listerine failed to prove significantly more active than placebo, although lower scores were determined. Equally interesting are the results obtained when GI was evaluated. The mushroom mouthwash showed a statistically significant advantage over both placebo and Listerine. This result may be attributed to the ability displayed by the mushroom mouthwash to reduce plaque deposition, but an anti-inflammatory activity or a sort of gingival cell protection by the mushroom extract may be suspected. Indeed, the LMM fraction of mushroom extract, at concentrations that did not affect the viability of $\mathrm{KB}$ gingival cells, is capable of modulating the expression of different genes induced by both live and heat-killed $P$. intermedia and A. naeslundii, thus supporting the hypothesis that this foodstuff extract can modulate the responses of gingival cells to periodontopathogens [20]. The effects of the mushroom mouthrinse on both PI and GI are reminiscent of those observed in oolong tea [11] and pomegranate [21] extract which significantly inhibit dental plaque deposition in volunteers but differ from those of nondialysable material of cranberry juice [12] for which no effect was detected on either gingival or plaque indices after a 42-day study. 


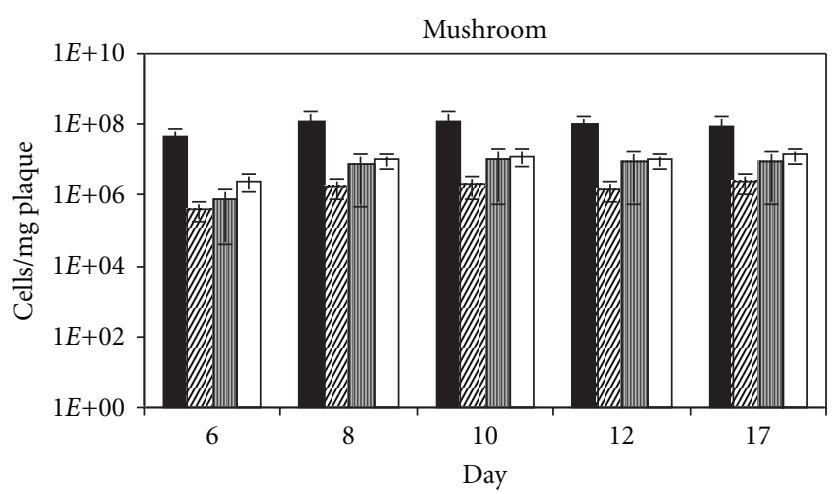

(a)

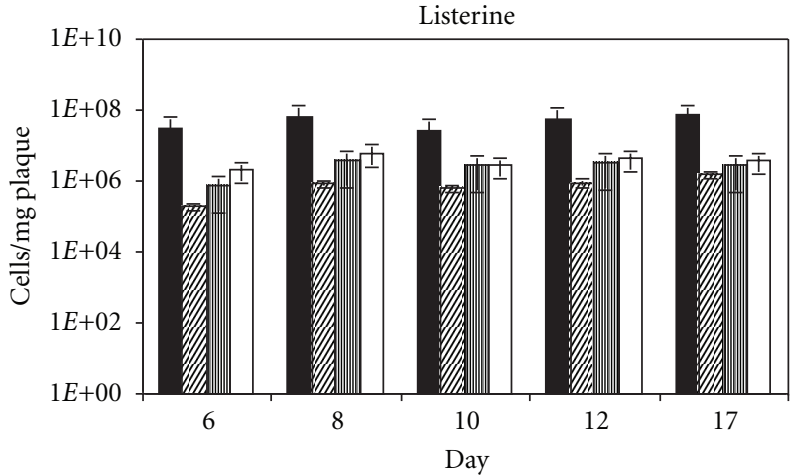

(b)

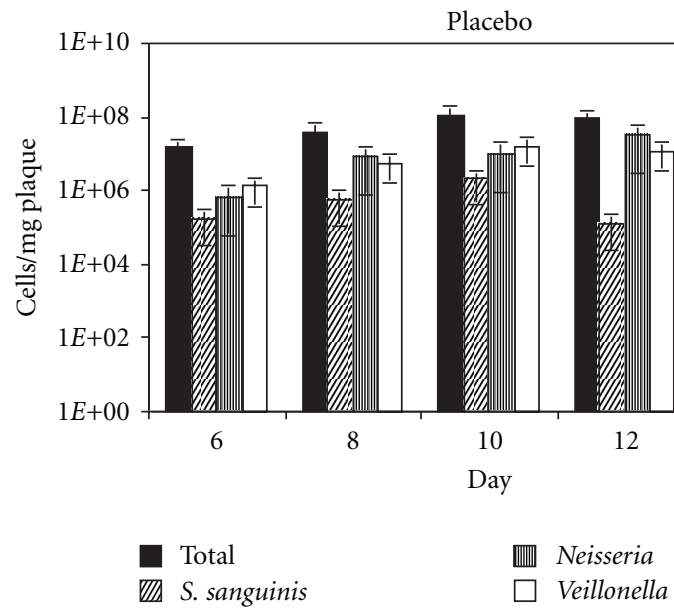

(c)

Figure 4: Numbers of each of the investigated taxa associated with oral health and total counts in dental plaque samples of the three cohorts enrolled in the study. Standard error bars represent the standard deviation $(n=30)$.

Chlorhexidine, which is capable of affecting both the clinical parameters PI and GI, is endowed, however, with a potent antibacterial activity $[22,23]$.

Although the mushroom mouthrinse is capable of significantly affecting clinical parameters, no significant reduction of total cell count, or of the counts of specific microbial components of dental plaque, was demonstrated. Some findings, however, deserve attention: as far as the effect on total plaque bacteria count is concerned, mushroom extract and Listerine may be equivalent in that they are capable of a certain degree of inhibition in comparison with the results of the placebo group, even if this activity failed to prove statistically significant. The partial activity against total plaque bacteria was studied in greater detail by analysing the effects on selected bacteria responsible for different oral conditions, such as the evolution towards gingivitis as indicated by the strict anaerobes $F$. nucleatum, $P$. intermedia, A. naeslundii and L. casei, caries following S. mutans, and the relationship between $N$. subflava, $V$. dispar, and S. sanguinis and oral health [19]. Against bacteria involved in gingivitis, mushroom extract and Listerine behaved similarly, inhibiting the increase in cell number in dental plaque of three out of four microorganisms tested (not including L. casei) to such an extent that the final number (day 17) is less than the half that of the placebo group. Although inhibition of the cell number increase is calculated as being about half the control value, it is worth noting that this inhibition is mainly attributable to strict oral anaerobes, whose role in the evolution of gingivitis towards periodontitis is fully ascertained [3, 4]. Against cariogenic S. mutans both mushroom and Listerine exerted a certain action, with cell counts on day 17 being roughly $50 \%$ lower than the control values. Also of interest are the results of the effects of the three mouthrinses on bacteria associated with oral health: a good candidate for formulating a mouthrinse should affect only potential oral pathogens, leaving that portion of oral microbiota associated with oral health unaltered. Mushroom extract seems to comply with this requirement in that it showed no effect mainly on gram-negatives (N. subflava and $V$. dispar) and only partial inhibition of S. sanguinis. From this point of view, the action of mushroom extract would appear to be superior to that of Listerine, with the latter being an inhibitor also of the microbiota associated with oral health. These results are in agreement with those obtained by Ciric et al. [24] who have tested the activity of the same mushroom fraction in an artificial mouth model (constant depth film fermentor) and shown that mushroom fraction lowered the numbers of 
some pathogenic taxa without affecting the taxa associated with oral health.

Although the microbiological results of the effect of the mushroom mouthrinse would appear to be in contrast with in vitro results which show antimicrobial, antiadhesive, antiplaque, and plaque disaggregating activities $[13,14]$, this incongruity may be explained by the fact that these activities were not relevant during the clinical trial or, alternatively, the experimental design was incapable of detecting them if present. Thus, on the basis of the promising effects on clinical parameters obtained with the mushroom mouthrinse, further studies conducted both in vitro and in humans are advisable.

The possibility of using mouthrinses containing active components of natural origin for daily oral hygiene may constitute a novel approach in order to alter biofilm formation on tooth and gum surfaces. Their potential for controlling dental caries and/or gingivitis/periodontitis warrants further investigation.

\section{Acknowledgment}

The research leading to these results has received funding from the European Union's Sixth Framework Programme (FP6) under contract FOOD-CT-2006-036210 (project NUTRIDENT).

\section{References}

[1] P. D. Marsh, "Dental plaque: biological significance of a biofilm and community life-style," Journal of Clinical Periodontology, vol. 32, supplement 6, pp. 7-15, 2005.

[2] N. H. Newman and M. Wilson, Dental Plaque Revisited: Oral Biofilms in Health and Diseases, Bioline, Cardiff, UK, 1999.

[3] P. D. Marsh and M. V. Martin, Oral Microbiology, Churchill Livingstone, Edinburgh, UK, 5th edition, 2009.

[4] R. J. Lamont, R. A. Burne, M. S. Lantz, and D. J. Leblanc, Oral Microbiology and Immunology, American Society for Microbiology Press, Washington, DC, USA, 2006.

[5] C. Signoretto, P. Canepari, C. Pruzzo, and G. Gazzani, "Anticaries and antiadhesive properties of food constituents and plant extracts and implications for oral health," in Food Constituents and Oral Health: Current Status and Future Prospects, M. Wilson, Ed., Woodhead Publishing Limited, Cambridge, UK, 2009.

[6] M. Elvin-Lewis and R. Steelman, "The anticariogenic effects of tea drinking among Dallas school children," Journal of Dental Research, vol. 65, no. 3, p. 198, 1986.

[7] C. Jones, K. Woods, G. Whittle, H. Worthington, and G. Taylor, "Sugar, drinks, deprivation and dental caries in 14year-old children in the north west of England in 1995," Community Dental Health, vol. 16, no. 2, pp. 68-71, 1999.

[8] C. Signoretto, G. Burlacchini, F. Bianchi, G. Cavalleri, and P. Canepari, "Differences in microbiological composition of saliva and dental plaque in subjects with different drinking habits," New Microbiologica, vol. 29, no. 4, pp. 293-302, 2006.

[9] C. Signoretto, F. Bianchi, G. Burlacchini, F. Sivieri, D. Spratt, and P. Canepari, "Drinking habits are associated with changes in the dental plaque microbial community," Journal of Clinical Microbiology, vol. 48, no. 2, pp. 347-356, 2010.
[10] M. Onisi, N. Shimura, C. Nakamura, and M. Sato, "A field test on the caries preventive effect of tea drinking," Journal of Dental Health, vol. 31, no. 1, pp. 13-19, 1981.

[11] T. Ooshima, T. Minami, W. Aono, Y. Tamura, and S. Hamada, "Reduction of dental plaque deposition in humans by oolong tea extract," Caries Research, vol. 28, no. 3, pp. 146-149, 1994.

[12] E. I. Weiss, A. Kozlovsky, D. Steinberg et al., "A high molecular mass cranberry constituent reduces mutans streptococci level in saliva and inhibits in vitro adhesion to hydroxyapatite," FEMS Microbiology Letters, vol. 232, no. 1, pp. 89-92, 2004.

[13] D. A. Spratt et al., "Evaluation of the beneficial oral health properties of plant and fungal extracts," Journal of Biomedicine and Biotechnology. In press.

[14] C. Signoretto, A. Marchi, A. Bertoncelli et al., "Effects of mushroom and chicory extracts on the physiology and shape of Prevotella intermedia, a periodontopathogenic bacterium," Journal of Biomedicine and Biotechnology. In press.

[15] M. Daglia, A. Papetti, D. Mascherpa et al., "Vegetable food components with potential activity on the development of microbial oral diseases," Journal of Biomedicine and Biotechnology. In press.

[16] S. Turesky, N. D. Gilmore, and I. Glickman, "Reduced plaque formation by the chloromethyl analogue of victamine C," Journal of Periodontology, vol. 41, no. 1, pp. 41-43, 1970.

[17] H. Loe and J. Silness, "Periodontal disease in pregnancy. I. Prevalence and severity," Acta Odontologica Scandinavica, vol. 21, no. 7, pp. 533-551, 1963.

[18] R. I. Griffiths, A. S. Whiteley, A. G. O'Donnell, and M. J. Bailey, "Rapid method for coextraction of DNA and RNA from natural environments for analysis of ribosomal DNAand rRNA-based microbial community composition," Applied and Environmental Microbiology, vol. 66, no. 12, pp. 54885491, 2000.

[19] L. Ciric, J. Pratten, M. Wilson, and D. Spratt, "Development of a novel multi-triplex qPCR method for the assessment of bacterial community structure in oral populations," Environmental Microbiology Reports, vol. 2, no. 6, pp. 770-774, 2010.

[20] L. Canesi, C. Borghi, M. Stauder et al., "Effects of fruit and vegetable low molecular mass fractions on gene expression in gingival cells challenged with Prevotella intermedia and Actinomyces naeslundii," Journal of Biomedicine and Biotechnology. In press.

[21] S. J. Bhadbhade, A. B. Acharya, S. V. Rodrigues, and S. L. Thakur, "The antiplaque efficacy of pomegranate mouthrinse," Quintessence International, vol. 42, no. 1, pp. 2936, 2011.

[22] D. H. Fine, "Chemical agents to prevent and regulate plaque development," Periodontology 2000, vol. 8, no. 1, pp. 87-107, 1995.

[23] B. M. Eley, "Antibacterial agents in the control of supragingival plaque-a review," British Dental Journal, vol. 186, no. 6, pp. 286-296, 1999.

[24] L. Ciric, A. Tymon, E. Zaura et al., "In vitro assesment of shiitake mushroom (Lentinula edodes) for its anti-gingivitis activity," Journal of Biomedicine and Biotechnology. In press. 

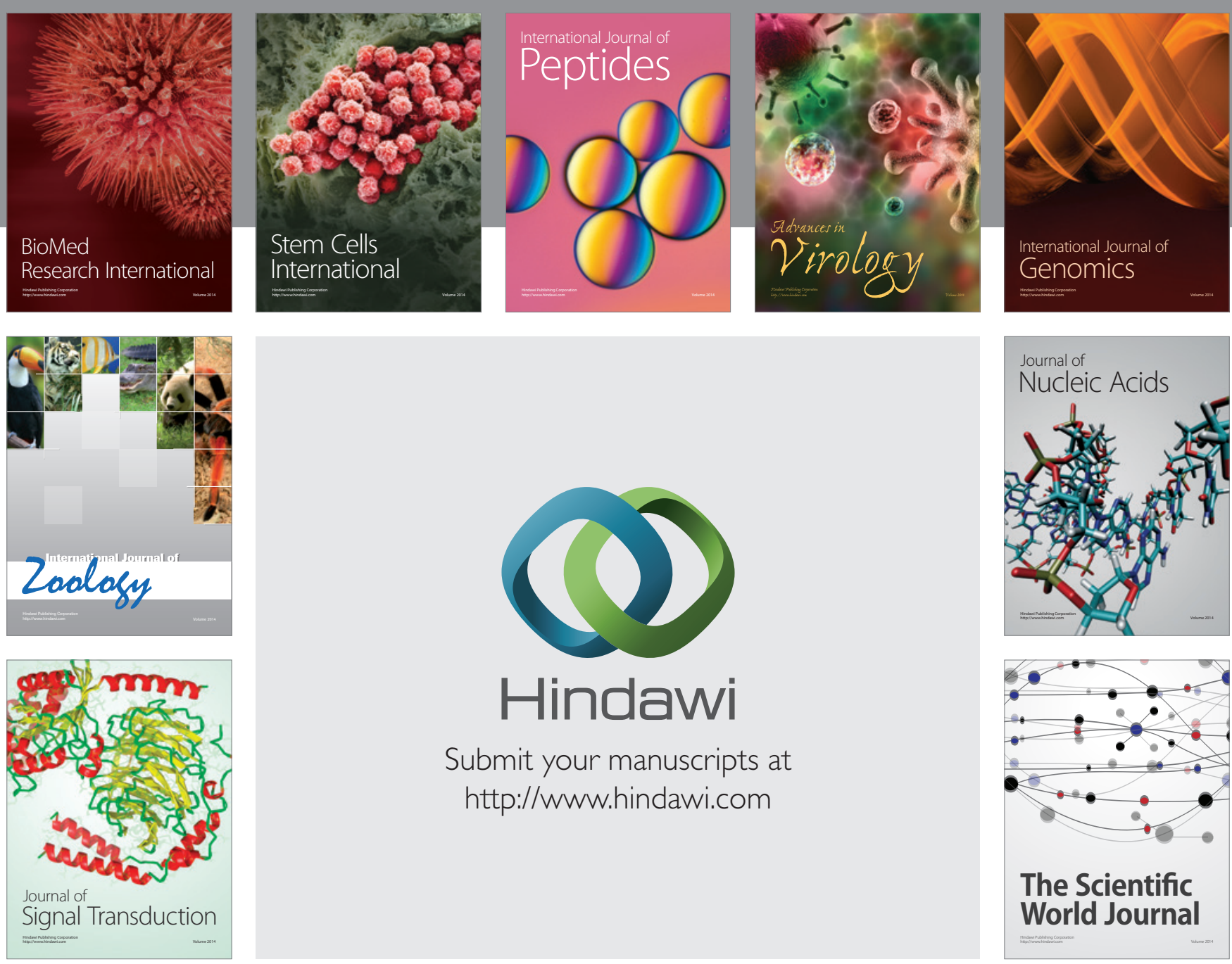

Submit your manuscripts at

http://www.hindawi.com
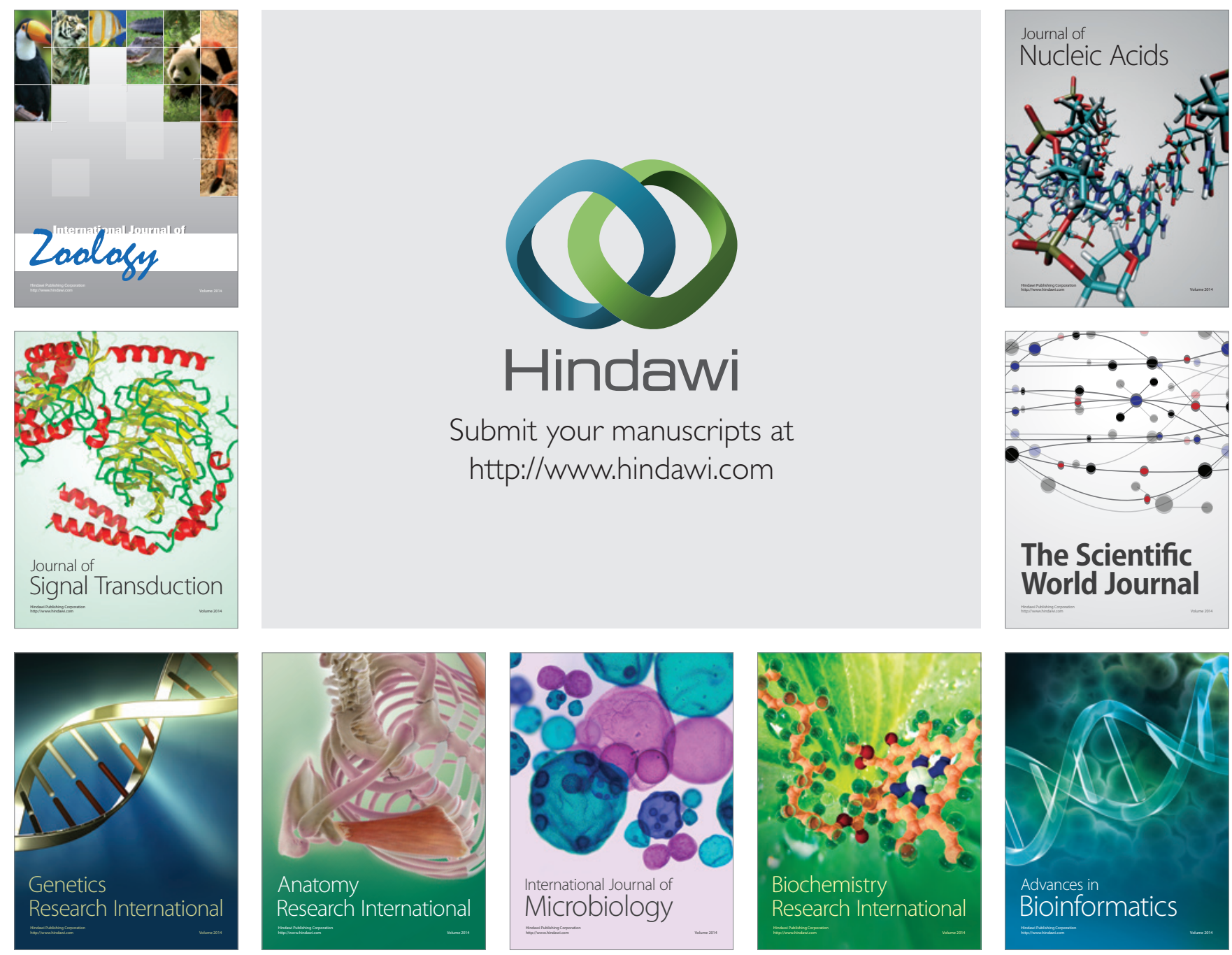

The Scientific World Journal
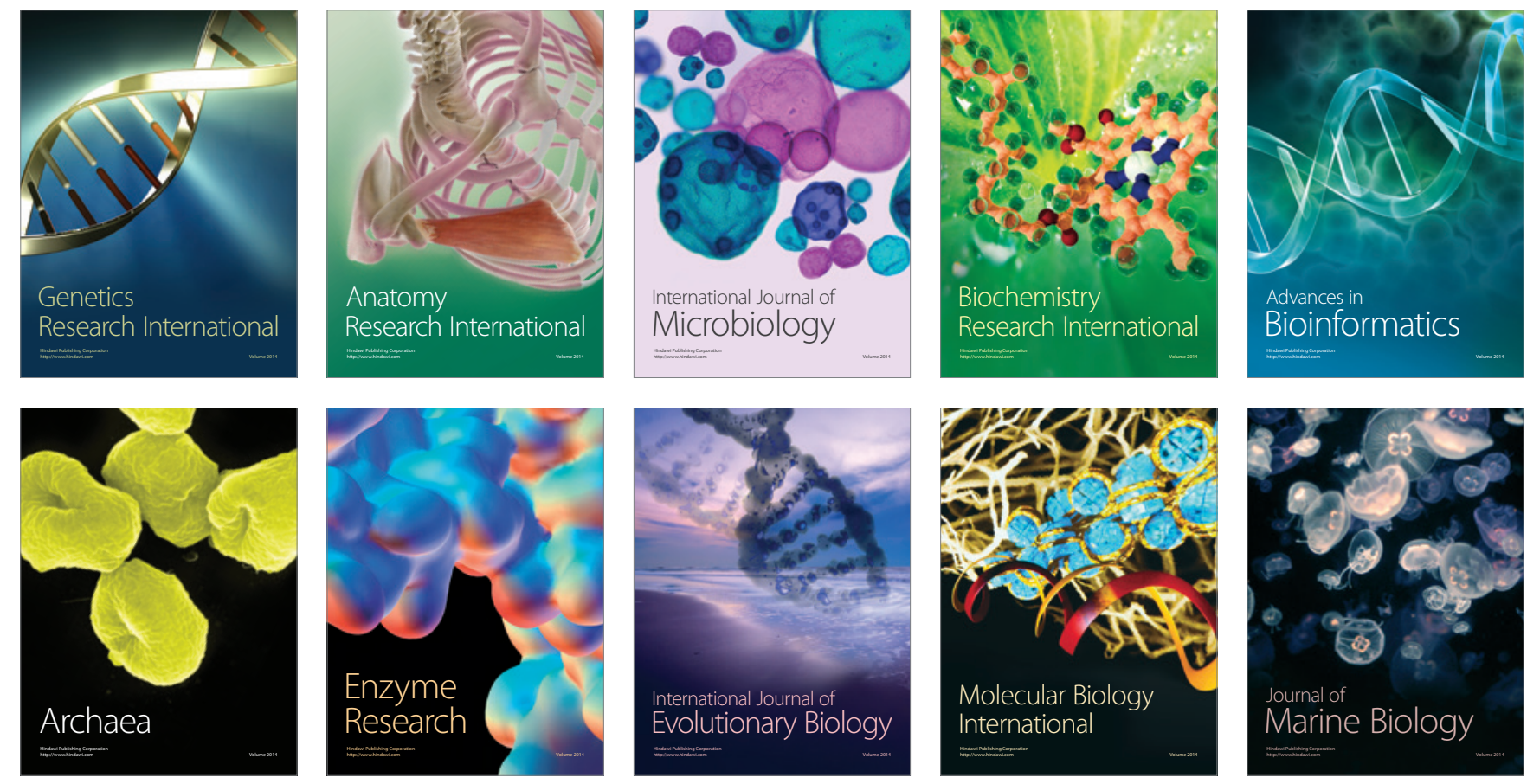